\title{
Diagnostic Approach Common Cases of Vitamins Toxicity in Children
} Dr. Raghba Shasho ${ }^{* *}$, Dr. Mohammad Shasho ${ }^{2}$

${ }^{1}$ Pediatric Specialist PHCC (Muaither Health Center), Doha, Qatar

${ }^{2}$ Orthopedic Surgeon, At Children's hospital of Syrian Arab Red Crescent, Aleppo, Syria

DOI: $10.36347 /$ sjams.2022.v10i01.001

| Received: 27.11.2021 | Accepted: 02.01.2022 | Published: 04.01.2022

*Corresponding author: Dr. Raghba Shasho

Pediatric Specialist PHCC (Muaither Health Center), Doha, Qatar

\section{Abstract}

Pediatric emergency departments have recorded visits by afraid parents who are worried that their child has been poisoned. Often, attendances of poisoning cases at emergency room after poison exposure is unnecessary, but sometimes for lifesaving. In young children especially among toddlers aged between one- and three-years poisoning is common accidentally while they explore their environment as part of their normal natural development, or deliberately in response to stress or underlying mental problems .Young children are at a high risk of accidental poisoning when they try to discover the world around them by playing with things - opening the cabinets, mother's handbag, imitating their parents or adult in actions especially if there is an elderly at home has one of geriatric diseases such as hypertension, diabetes mullites ... and taking medication contiguously so an accidental poisoning occurs as a trial from child to learn who ingest that. The child is detected by parents or caregiver by chance while playing with a packet of tablets or with some of them in mouth. Deliberately poisoning usually happens in adolescent as attempting self-harm or suicide purpose by ingested large quantities of tablets than young children. Poisoning may be presents because of error doses which is prescribed by health professionals by wrong.

Keywords: Diagnostic Vitamins Toxicity Children.

Copyright (C) 2022 The Author(s): This is an open-access article distributed under the terms of the Creative Commons Attribution 4.0 International License (CC BY-NC 4.0) which permits unrestricted use, distribution, and reproduction in any medium for non-commercial use provided the original author and source are credited.

\section{Definition}

Toxicity means the condition of the substance and the level to which a chemical substance can be harmful and cause damage to body especially if it is exceeded the highest recommended doses. Ingested substance may be medicines (analgesic, vitamins, antibiotic, oral contraceptive, opioids) or household products (cleaning liquid products, strong bleach).

Toxic exposures occur frequently in children throughout the world. Common types of pediatric poisoning present in creeping or walking children by ingestion medication accidently as explorer the surrounding environment, or the other side in older children and adolescent the witnessed ingestion is so common with a child who found handle a packet of tablets or a container of medication that look like candy.

Vitamins are essential nutrients found in foods. They perform specific and vital functions in a variety of body systems and are crucial for maintaining optimal health. The two different types of vitamins are fatsoluble vitamins and water-soluble vitamins. Fat- soluble vitamins - vitamins $\mathrm{A}, \mathrm{D}, \mathrm{E}$ and $\mathrm{K}$ - dissolve in fat before they are absorbed in the bloodstream to carry out their functions. Excesses of these vitamins are stored in the liver and are not needed every day in the diet. In contrast, water-soluble vitamins dissolve in water and are not stored by the body. Since they are eliminated in urine, we require a continuous daily supply in our diet. The water-soluble vitamins include the vitamin B-complex group and vitamin $\mathrm{C}$ [1].

Vit A: Vitamin A is a fat-soluble vitamin necessary for health, deficiency of which can cause disorders of vision, skin, bone, and immunity.

Individuals who ingest large amounts of provitamin A (from plant sources) may develop yellowtinged skin (carotenemia) without developing vitamin A toxicity. Carotenemia is particularly common among infants and toddlers who are eating large amounts of pureed vegetables (particularly carrots and green leafy vegetables) and may be initially confused with jaundice. The skin discoloration resolves spontaneously if the intake of these foods is reduced [2]. 
Vitamin A toxicity presents with dry, scaly skin with areas of desquamation and fissuring of the lips. Other symptoms include headache, fatigue, anorexia, nausea, vomiting, blurred vision, pseudotumor cerebri, myalgias, and arthralgias. Recommended daily intake of vitamin A is between 100 and $5000 \mathrm{IU}$. In children, however, hypervitaminosis develops quickly and usually resolves quickly [3].

Acute vitamin A toxicity usually appears after more than $500 \mathrm{mg} /$ day in adults, $100 \mathrm{mg} /$ day in children or $30 \mathrm{mg}$ /day in infants [4].

Acute toxicity: In infants under six months of age, as little as 20,000 international units (6000 micrograms) daily, given briefly (eg, for one month or less), may produce toxic effects [2].

Vitamin A toxicity is increased osteoclast activity and bone resorption; this results in decreased bone density, skeletal hyperostosis and hypercalcemia, with low or normal parathyroid hormone concentrations and occasionally metastatic calcifications.

Vitamin A toxicity is an important diagnosis to consider in patients with hypercalcemia [5]. Chronic toxicity can appear after a long-term intake of 10 $\mathrm{mg}$ /day of vitamin A for several months in adults and $7.5-15 \mathrm{mg} / \mathrm{day}$ in children. In general, toxicity is uncommon in intakes lower than $30 \mathrm{mg}$ /day $(25,000-$ 30,000 IU/day)[4].

Chronic toxicity: Some toxic effects of vitamin A also have been observed in infants fed large amounts of chicken liver daily for one month or longer; each serving contained 36,000 international units $(11,000$ micrograms retinol), which is more than 20 times the RDA for this age group [6].

The recommended daily allowance for vitamin $\mathrm{A}$ is 300 to $700 \mu \mathrm{g}$ for children [7]. (Where 1 microgram retinol $=3.3$ international units)

Vit B: The B-vitamins comprise a group of eight water soluble vitamins that perform essential, closely inter-related roles in cellular functioning, acting as co-enzymes in a vast array of catabolic and anabolic enzymatic reactions $[\mathbf{8}]$.

B-complex Vitamins Eight of the watersoluble vitamins are known as the vitamin B-complex group: thiamin (vitamin B1), riboflavin (vitamin B2), niacin (vitamin B3), vitamin B6 (pyridoxine), folate (folic acid), vitamin B12, biotin and pantothenic acid. The $\mathrm{B}$ vitamins are widely distributed in foods [8].

\section{Thiamin (vitamin B1):[6]}

Sources: Thiamine is primarily found in foods such as yeast, legumes, pork, brown rice, and cereals made from whole grains. The thiamine molecule is denatured at high $\mathrm{pH}$ and high temperatures. Then, cooking, baking, and canning of some foods as well as pasteurization can destroy thiamine. Milk products, fruits, and vegetables are poor sources of thiamine.

Toxicity: No syndrome of excess thiamine has been identified. It is believed that toxic levels are unlikely because the kidneys can rapidly clear almost all excess thiamine and because (like most watersoluble vitamins) thiamine is not stored. The biologic half-life of thiamine in humans is approximately 10 to 20 days.

Requirements: for thiamine for different lifestage groups is 0.5 to $0.9 \mathrm{mg} / \mathrm{day}$ for children. Riboflavin (vitamin B2): [6]

Sources: Riboflavin is supplied in many foods, including milk, eggs, meats, fish, green vegetables, yeast, and enriched foods (fortified cereals and breads).

Toxicity: No adverse effects have been reported after ingestion of high doses of riboflavin.

Requirements: The United States recommended dietary allowance (RDA) for riboflavin is 0.5 to $0.9 \mathrm{mg} /$ day in children.

\section{Niacin (vitamin B3)}

Requirements: Niacin is dosed as a "niacin equivalent" (NE), in which $1 \mathrm{NE}$ is equal to $1 \mathrm{mg}$ of niacin, or $60 \mathrm{mg}$ of dietary tryptophan. The United States recommended dietary allowance (RDA) for niacin is 6 to $12 \mathrm{mg} /$ day in children [6].

The UL is 10 and $35 \mathrm{mg}$ per day for children and adults, respectively. These limits are based on the appearance of nonlife-threatening symptoms of flushing. Niacin toxicity presentenced by flushing pruritus -hives-vomiting and elevated liver enzymes.

\section{B6 (pyridoxine) [6]}

Sources: Pyridoxine and pyridoxamine are predominantly found in plant foods; pyridoxal is most derived from animal foods. Meats, whole grains, vegetables, and nuts are the best sources. Cooking, food processing, and storage can reduce vitamin B6 availability by 10 to 50 percent.

Requirements: The recommended dietary allowance (RDA) of vitamin B6 ranges from 0.5 to 1 $\mathrm{mg} /$ day in children.

Toxicity presentation: Neuropathy photosensitivity. Folate (folic acid) B9: Folic acid is a water-soluble vitamin included in the B-vitamin group, also known as vitamin $\mathrm{B}_{9}$, and it is normally found in various vegetables. 
Folic acid is also fundamental for nucleic acid synthesis, amino acid metabolism and appropriate cellular replication. Daily recommended intake is between 110 and $320 \mu \mathrm{g} / \mathrm{d}$ in breastfed infants and adolescents [9].

Vitamin B12 (Cobalamin): Vitamin $\mathrm{B}_{12}$ is a water-soluble vitamin that can be naturally found in animal products. It is involved in DNA and $\mathrm{Hb}$ synthesis and is fundamental for proper neurological function, in order to maintain low levels of homocysteine to prevent damage at the endothelial level, and it acts as a cofactor in numerous metabolic pathways. Recommended intake for children varies between $0.7 \mu \mathrm{g} / \mathrm{d}$ in toddlers and $2 \mu \mathrm{g} / \mathrm{d}$ during adolescence [9]. Toxicity: No adverse effects have been demonstrated with high vitamin B12 intake.

\section{Biotin: [6]}

Sources: Biotin can be found in a variety of plants but is found in highest levels in the liver, egg yolk, soybean products, and yeast. It is bound to proteins in foods and becomes bioavailable after it is released through the action of the enzyme biotinidase.

Toxicity: No toxicity of excess biotin intake has been described.

Requirements - the recommended intake for biotin is expressed as adequate intake (AI) is 8 to 12 $\mathrm{mcg} /$ day for children

\section{VITAMIN B5 (PANTOTHENIC ACID) [6]}

Sources: The major dietary sources of pantothenic acid are egg yolk, liver, kidney, broccoli, and milk. The main dietary source of pantothenic acid is in the form of coenzyme A $(\mathrm{CoA})$. Pantothenic acid is also produced by bacteria in the colon

Toxicity: There is no known toxicity for pantothenic acid. Excess intake is excreted by the kidneys.

Requirements: The recommended intake for pantothenic acid is expressed as adequate intake AI is 2 to $4 \mathrm{mg} /$ day for children

Vit C: Vitamin C is a water-soluble vitamin, antioxidant, and essential co-factor for collagen biosynthesis, carnitine and catecholamine metabolism, and dietary iron absorption. Humans are unable to synthesize vitamin $\mathrm{C}$, so they can only obtain it through dietary intake of fruits and vegetables. Citrus fruits, berries, tomatoes, potatoes, and green leafy vegetables are excellent sources of vitamin $\mathrm{C}$. Although most vitamin $\mathrm{C}$ is completely absorbed in the small intestine, the percentage of absorbed vitamin $\mathrm{C}$ decreases as intraluminal concentrations increase. Proline residues on procollagen require vitamin $\mathrm{C}$ for hydroxylation, making it necessary for the triple-helix formation of mature collagen [10].

Vit C toxicity presented as complaint of diarrhea and abdominal bloating. High vitamin $\mathrm{C}$ intakes also have the potential to increase urinary oxalate and uric acid excretion, which could contribute to the formation of kidney stones, especially in individuals with renal disorders [11].

Requirements: It ranges from 15 to $45 \mathrm{mg} /$ day in children [2]. Mean intakes for children and adolescents aged 1-18 years range from $75.6 \mathrm{mg} /$ day to $100 \mathrm{mg} /$ day [11].

Iron: Iron is found in many over the counter (OTC) multivitamins. Iron toxicity from intentional or accidental ingestion is a common poisoning. Children of pregnant mothers or breastfeeding mothers are at a high risk of iron toxicity due to it is available for them, so it is too easy to get it.

Accidental ingestions are more common in children less than 6 years. In addition, iron toxicity may also develop after multiple blood transfusions for a chronic disorder like thalassemia, sickle cell, and hematological cancers [12].

Ingestions doses of less than $20 \mathrm{mg} / \mathrm{kg}$ of elemental iron typically do not result in symptoms while toxic levels usually occur in ingested doses of greater than $40 \mathrm{mg} / \mathrm{kg}$ elemental iron. For life threating levels with ingestion doses of greater than $60 \mathrm{mg} / \mathrm{kg}$ elemental iron.

Serum iron level peaks should be obtained at 4 to 6 hours post-ingestion. Approximately $10 \%$ of ingested iron is absorbed from the intestine and is subsequently bound to transferrin. In overdoses, total iron bending capacity is saturated and free ferric iron $\left(\mathrm{Fe}^{+3}\right)$ is increase and leads to production of free radical which results in local tissue injury.

Excess iron will circulate in the blood as free iron, which is directly toxic to target organs [14].

Iron is directly corrosive to the gastrointestinal mucosa and may cause life threating hemorrhage, necrosis, or both.

The clinical course of iron toxicity is divided into five stages. The progression from stage to stage may be very rapid. It should be noted that patients can skip any of these stages.

1. During the first stage $(30 \mathrm{~min}$ to 6 hours after ingestion), local corrosive effect on gut mucosa leads to complaint of nonspecific gastrointestinal (GI) symptoms such as abdominal pain, nausea, vomiting, diarrhea, hematemesis, and melena. 
Raghba Shasho \& Mohammad Shasho; Sch J App Med Sci, Jan, 2022; 10(1): 1-8

If a patient does not experience stage 1 within 6 hours of the ingestion that's mean the amount of iron consumed is likely not significant.

2. The second stage (6 to 12 hours) referred to the latent stage patient is often asymptomatic and the previous gastrointestinal symptoms which appear at the 1 st stage will improve.

3. The third stage ( 12 to 48 hours) is characterized by the recurrence of GI symptoms, hypoperfusion, shock, and metabolic acidosis, coma and acute hepatic dysfunction with hypoglycemia and coagulopathy.

4. The fourth stage ( 2 to 6 weeks after ingestion) is by an elevation of aminotransferase levels and possible progression to hepatic failure.

5. The fifth stage ( 2 to 4 weeks later) represents the consequences of the healing of the injured GI mucosa including pyloric or proximal bowel scarring and obstruction.

Determination of the iron toxicity stage should be based on symptoms and clinical manifestations and not on time of ingestion [12].

Vit D: Vitamin D is a fat-soluble pro-hormone primarily synthesized in skin by sun-light exposure. This vitamin has a crucial role in the health of the musculoskeletal system. Furthermore, vitamin D has beneficial effects on cardiovascular, respiratory, and immune systems [13].

An excess of Vitamin D is generally associated with unnecessary or inappropriate doses of Vitamin D and long-term outpatient prescribing, especially nowadays using vit $\mathrm{D}$ is increasing as prophylactic and treatment purpose in the outbreak of COVID-19 infection.

Fortified foods such as milk or breakfast cereals are good sources of dietary vitamin D2. Vitamin D3 is synthesized in the skin by exposure to sun light. Both are converted to 25 -hydroxyvitamin D (25- OH - D calcitriol) in the liver then transported to the kidney to form 1, 25- dihydroxy vitamin D ( 1-25- OH- D , calcitriol ).

Vitamin D concentration is measured by 25 hydroxy vitamin D level because it has longer half-life compared to $1,25 \mathrm{OH}$ vitamin $\mathrm{D}$ [13].

Based on guidelines by the Institute of Medicine, recommended dietary allowances for vitamin $\mathrm{D}$ are $600 \mathrm{IU} /$ day for those aged between 1 and 70 years and $800 \mathrm{IU} /$ day for those aged 71 and above. For babies below one year of age, the American Academy of Pediatrics recommends 400 IU/day. These dietary recommendations should result in serum 25hydroxyvitamin D levels of $\geq 20 \mathrm{ng} / \mathrm{mL}$. At the other end of the scale, the Food and Nutrition Board has set upper intake levels of vitamin D at $2000 \mathrm{IU} /$ day (50 $\mu \mathrm{g} /$ day). Doses higher than 50,000 IU/day raise serum concentrations above $150 \mathrm{ng} / \mathrm{mL}$, leading to hypercalcemia [14].

The upper limit for children younger than 1 year has been reported to be $1000 \mathrm{IU} /$ day for long-term Vitamin D therapy, and 2000 IU/day for children over 1 year. Pediatric toxic threshold doses are not clearly defined, and serum $25(\mathrm{OH}) \mathrm{D}$ concentration is up to $140 \mathrm{ng} / \mathrm{it}$ is considered harmful if it is low [15].

A definite amount of vitamin $\mathrm{D}$ ingestion to cause toxicity has not been elucidated. Although maximum tolerable ingested dose is various in different age groups, maximum tolerable and safe dose of vitamin $\mathrm{D}$ is $1000 \mathrm{IU} /$ day in infants younger than 6 months, $1500 \mathrm{IU} /$ day in children older than 6 months, and $10000 \mathrm{IU} /$ day in adults [15].

The acute toxic dose for vitamin D has not been established. The chronic toxic dose is more than $50,000 \mathrm{IU} /$ day in adults. In infants younger than 6 months, 1,000 IU/day may be considered unsafe. However, a wide variance in potential toxicity exists for vitamin D [16].

Although rare, cases of vitamin D intoxication that present with dramatic life-threatening symptoms still occur in children. Moreover, recent studies in infants raise a potential need for monitoring vitamin D levels when doses at or above the currently recommended upper range are used. Further studies are needed to clarify these findings. The Drugs and Therapeutics Committee of the Pediatric Endocrine Society suggests obtaining serum 25 -hydroxyvitamin D levels in infants and children who receive long-term vitamin D supplementation at or above the upper-level intake that is currently recommended [17].

Vitamin D toxicity may indicate symptoms such as hypercalcemia symptoms (polyuria, polydipsia, nausea, vomiting, loss of appetite, constipation), hyperphosphatemia, nephrolithiasis, nephron calcinosis, ectopic calcification of soft tissues (kidney and lung), depression.

Vitamin D is characterized by hypercalciuria and hypocalcemia-associated nephrocalcinosis and $25(\mathrm{OH})$ D vitamin (>150 ng/ $\mathrm{mL}$ ) parathyroid hormone suppression [15].

Vit E: Vitamin E is a fat-soluble compound and an antioxidant and protects cell membranes from oxidation and destruction. It is the collective term for four tocopherols $(\alpha-, \beta-, \gamma-$, and $\delta$-tocopherols $)$ and four tocotrienols ( $\alpha-, \beta-, \gamma-$, and $\delta$-tocotrienols) found in food, with antioxidant activities, but cannot be interconverted, and activities, but cannot be interconverted, and -tocopherol meets the human vitamin E requirement [18]. 
Vitamin $\mathrm{E}$ is found in various foods and oils. Nuts, seeds, and vegetable oils contain high amounts of alpha-tocopherol, and significant amounts are also available in green leafy vegetables and fortified cereals [19]. In children, the recommended daily allowance rises from $6 \mathrm{mg}$ at 1 to 3 years of age to $15 \mathrm{mg}$ by 14 to 18 years [18].

Large oral supplements of vitamin $\mathrm{E}$ were associated with impaired response to iron in anemia impaired neutrophil function -and sepsis in premature infants.

Vit $\mathrm{K}$ is required for the formation of prothrombin and some other factors of blood clotting in the liver. Also, it plays role in metabolism of the bone. A form of vitamin $\mathrm{K}$ is produced by bacteria in colon and can be used to some degree [20].

Vitamin K1 is found mainly in green leafy vegetables such as kale, spinach, and broccoli while vitamin $\mathrm{K} 2$ is found in liver, milk, cheese, and fermented soy products such as Natto. Menadione is a chemically synthesized derivative used for animal feed [21].

Toxicity: Vitamin K toxicity is extremely rare. When toxicity does occur, it manifests with signs of jaundice, hyperbilirubinemia, hemolytic anemia, and kernicterus in infants. Vitamin $\mathrm{K}$ toxicity is very rare, and a tolerable upper limit for consumption has not been defined. Menadione, one of the precursors of vitamin $\mathrm{K}$, can cause hemolytic anemia, hyperbilirubinemia, jaundice, and kernicterus in infants. Menadione was used in pre-mature or low-birth-weight newborns and precipitated kernicterus only in high doses but was not widely available [22].

The Adequate intake in children ranges from 2 micrograms daily in young infants to 75 micrograms daily in adolescent boys. The adequate intake in infants assumes that infants also receive vitamin $\mathrm{K}$ prophylaxis at birth [23].

\section{An approach to the evaluation and management of vitamins toxicity cases}

History: Clinical history is the key to identify the potential causes of vitamins poisoning. Parents/ child / adolescent should be questioned to identify the possible poisoned agents.

Physical exam: Physical examination stills the cornerstone to evaluate the presenting complaint. A careful physical exam should be completed to collect the clinical signs which will be helpful to identify the poisoned agent if the taken substance is unknown. An overdose or poison ingestion in a child is a clinical diagnosis supported by laboratory findings and imaging studies.
Diagnostic evaluation: Initially we should consider the severity of toxicity - the time since ingestion and the reported dose ingested. At the first stage of ingestion or in case of ingested small amounts of medication, patient may be asymptomatic with stable condition and normal findings in physical examination, so no need for other investigations. While in presentation with non - specific symptoms such as vomiting, diarrhea it is so important to monitor electrolyte levels, and assess anion gap.

\section{Laboratory Studies [24]}

Vitamin A: Laboratory workup to evaluate vit A toxicity include the following: Complete blood count (CBC) to rule out leukopenia, calcium, glucose, serum electrolytes If vomiting or diarrhea is present and liver function tests (LFTs). It is so necessary to obtain serum retinol concentrations in high raised levels of Vit A.

The reference range for vitamin $\mathrm{A}$ is 20-60 $\mathrm{mcg} / \mathrm{dL}$, and a toxic level is higher than $60-100$ $\mathrm{mcg} / \mathrm{dL}$.

For serum carotene, the normal range is 50$300 \mathrm{mcg} / \mathrm{dL}$. Carotene levels reflects dietary intake of vitamin $\mathrm{A}$.

A lumbar puncture may be indicated to rule out increased intracranial pressure in patients with vitamin A toxicity.

\section{B Vitamins}

Recommended laboratory studies in patients with possible B vitamin toxicity are as follows:

- Vitamins B1, B2, and B12 - These require no specific laboratory tests.

- Vitamin B3 - Perform LFTs. Uric acid may be increased, leading to gouty arthritis. The glucose level is occasionally elevated.

- Vitamin B6 - Vitamin B6 toxicity does not require laboratory or other tests. Lumbar puncture may be considered to rule out other causes if the patient has a peripheral neuropathy.

\section{Vitamin C}

Perform urinalysis to rule out uricosuria. False-negative test results for glucosuria are possible. Also perform renal function tests. Serum iron levels should also be measured because vitamin $\mathrm{C}$ enhances iron absorption.

\section{Vitamin D}

Obtaining calcium levels is mandatory; they are usually above $11 \mathrm{mg} / \mathrm{dL}$ but may be much higher. Phosphate levels may increase with calcium.

Kidney function tests (ie, blood urea nitrogen [BUN] and creatine tests, as well as urinalysis) are necessary to rule out possible kidney damage from hypercalciuria. 
Vitamin E

Measure PT activated partial thromboplastin time (aPTT), and bleeding times, especially if any evidence of bruising or bleeding is present. Platelet aggregation studies may be performed if bleeding time results are abnormal.

Monitor PT in patients who are taking anticoagulants concurrently with vitamin $\mathrm{E}$ or in patients suggested to have vitamin $\mathrm{K}$ deficiency while taking vitamin E, because the PT may be elevated.

The plasma concentration of alpha tocopherol (normal, 6-14 $\mathrm{mcg} / \mathrm{mL}$ ) can be measured to confirm that high levels of vitamin $\mathrm{E}$ are in the blood. oral anticoagulants

Vitamin K: Measure PT if the patient is taking

Iron: monitoring the followings: Serum electrolytes if gastrointestinal symptoms present - Liver function tests (LFTs) to monitor liver function Complete blood count (CBC) - For anemia, leukopenia, or thrombocytopenia, suggest signs of anemia due to gastrointestinal hemorrhage from iron toxicity. Serial serum iron concentrations are useful in monitoring asymptomatic patients or for patients who ingested slow-release enteric coated formulations.

In addition to laboratory studies, imaging and electrocardiographic studies can be used in the assessment of patients with vitamin toxicity [24].

\section{Imaging studies can be as diagnostic tool as follows}

*Iron tables are radio-opaque so they should be apparent on $\mathrm{X}$ - Ray in the gastrointestinal tract while liquid preparations and children's chewable tablets and multivitamins are not often visible on $\mathrm{X}-$ ray.

- Skeletal radiography - For calcifications in chronic vitamin A and vitamin D toxicity

- Hand radiography - For periosteal calcifications

- Helical computed tomography (CT) scanning and urography - Obtain a helical CT scan or an intravenous urogram (IVU) for suspected nephrolithiasis (ie, oxalate stones) in patients with vitamin $\mathrm{C}$ toxicity

- CT scanning of the brain - In the presence of neurologic abnormalities, perform a CT scan of the brain, without contrast, only if the prothrombin time (PT) is significantly prolonged and the patient has either a decreased level of consciousness or a focal neurologic deficit
- Kidneys, ureters, bladder (KUB) film - Indicated for suspected toxicity from iron-containing pills

- Bone mineral density testing - to evaluate the effect of long-term vitamin A intoxication on reducing bone density and causing osteoporosis [24]

*Obtain an electrocardiogram (ECG) to evaluate for effects of hypercalcemia in patients with vitamin D toxicity.

\section{Treatment $\backslash$ Management [24]}

Toxicity of vit A: Symptoms of vitamin A toxicity usually resolve after stopping vitamin A and instituting supportive therapy. The pigmentation of carotenemia usually disappears by exclusion of carrots from the diet.

Patients with increased intracranial pressure may need therapeutic lumbar punctures or further treatment with medications such as diuretics and mannitol.

\section{Patients with symptomatic hypercalcemia require the following}

- Close monitoring

- Treatment with intravenous fluids and diuretics

- Additional therapy, including pamidronate, calcitonin, corticosteroids, or mithramycin

- Discontinuation of vitamin A

Other Vitamins: Vitamins K, B1, B2, B6, B12, and C, and folate

These usually require only supportive measures.

Toxicity of vit D: Treatment options for vitamin $\mathrm{D}$ intoxication in children currently include discontinuation of vitamin $\mathrm{D}$ intake, intravenous hydration $(\mathrm{IH})$ with normal saline, administration of $\mathrm{F}$, glucocorticoids, calcitonin, alendronate, pamidronate and hemodialysis [25].

Prednisolone is less effective in the treatment of children with severe hypercalcemia secondary to vitamin $\mathrm{D}$ intoxication and timely implementation of other treatment regimens should be considered [26].

Vitamin $\mathrm{D}$ intoxication resulting in hypercalcemia suggests that cases with serum calcium levels below $12 \mathrm{mg} / \mathrm{dL}$ can be treated without prednisolone and bisphosphonates. Prednisolone treatment is less effective in the treatment of children with "severe" hypercalcemia (serum calcium levels above $14 \mathrm{mg} / \mathrm{dL}$ ) and prompt implementation of pamidronate should be considered [26]. 
Toxicity of Vit E

Management of vitamin E toxicity consists of discontinuing vitamin E supplements and
monitoring the PT if bleeding complications develop.
Vitamin K replacement through the oral or subcutaneous route should reduce the elevated PT
and decrease the risk of bleeding in patients who are taking anticoagulants or who have vitamin
K deficiency.

Vitamin B3: Provide supportive care as needed.

Toxicity of Iron: The following is a formula used to calculate the amount of ingested iron for a 10$\mathrm{kg}$ child who consumed 10 tablets of $320 \mathrm{mg}$ ferrous gluconate ( $12 \%$ elemental iron per tablet):

$320 \mathrm{mg}$ ferrous gluconate $\times 0.12$ elemental iron per tablet $=38.4 \mathrm{mg}$ elemental iron per tablet $\times 10$ tablets $=384 \mathrm{mg} / 10 \mathrm{~kg}=38.4 \mathrm{mg} / \mathrm{kg}$.

Carbonyl iron and iron polysaccharide complex are nonionic forms of iron that have less toxicity than ferrous salts [26].

Management will depend on the symptoms which patient presented with and at which stage of iron toxicity stages belongs to.

Patients who remain asymptomatic 4 to 6 hours after ingestion or those who have not ingested a potentially toxic amount do not require any treatment for iron toxicity.

Patients who presented with GI symptoms (1 st stage) that resolve after a short period of time and have normal vital signs require supportive care and an observation period, as it may represent the second stage of iron toxicity.

Patients who are symptomatic or demonstrate signs of hemodynamic instability require aggressive management and admission to an intensive care unit. It is so important to consider the following steps of algorithm treatment in iron toxicity:

1. Correct hypovolemia and hypoperfusion by IV fluids.

2. Deferoxamine, is a specific iron chelator, excreted by the kidneys. The indication for using it include metabolic acidosis (but metabolic alkalosis is not ), sever symptoms, and serum level greater than 500 $\mathrm{mcg} / \mathrm{dl}$ or greater than $350 \mathrm{mcg} / \mathrm{dl}$ if the bill presented on $\mathrm{X}$ ray.

It is administered as a continuous infusion at $15 \mathrm{mg} / \mathrm{kg} / \mathrm{hr}$ for up to 24 hours increasing the dose as needed with a maximum dose up $45 \mathrm{mg} / \mathrm{kg} / \mathrm{hr}$. Infusion rate should be decreased if hypotension occurs. Clinical recovery guides the termination of deferoxamine therapy, but the duration of therapy is typically 24 hours. Consultation with a toxicologist is highly recommended.

Treatment with intravenous deferoxamine should be considered for iron levels >500 $\mu \mathrm{g} / \mathrm{dL}$, significant acidosis, or ongoing symptoms [27].

3. Coagulopathy can be corrected with vitamin K (5 to $10 \mathrm{mg}$ subcutaneously) and fresh frozen plasma (10 to $25 \mathrm{~mL} / \mathrm{kg}$ in adults; $10 \mathrm{~mL} / \mathrm{kg}$ in children).

4. Endoscopic removal of the tablets or whole bowel irrigation (EX. polyethylene glycol at $10-15 \mathrm{ml} /$ $\mathrm{kg} / \mathrm{h}$ ) would be appropriate treatment options if abdominal x-ray demonstrates a large number of visible pills in the stomach. For most cases, the risks of gastric lavage outweigh the benefits.

5. Activated charcoal will not absorb iron and studies have not shown gastric lavage to improve outcomes. Using sodium bicarbonate, without an associated metabolic acidosis is not a therapy for iron toxicity.

Patients with GI symptoms or evidence of dehydration should be admitted. Patients receiving deferoxamine treatment should be admitted as well. Intensive care unit admission is indicated for patients presenting with coma, shock, metabolic acidosis, or iron levels over $1000 \mathrm{mg} / \mathrm{dL}$. Psychiatric referral is indicated for patients with an intentional overdose. Patients can be safely discharged if they are asymptomatic after a 6 to 12-hour observation period and have a negative radiograph, or if they have mild GI symptoms that resolve without metabolic acidosis and serum iron level under $350 \mathrm{mg} / \mathrm{dL}$ after a 6-to-12-hour observation period [26].

Patients with iron bezoars may require surgery. In severe cases, hemodialysis can also be effective [26]. Ipecac-induced emesis is not recommended. This is especially true in iron ingestion, as GI distress is an early finding in iron poisoning and is present in all potentially serious ingestions, and ipecac-induced vomiting may cloud the clinical picture [27].

Gastric lavage is not recommended because iron tablets are relatively large and become sticky in gastric fluid, making lavage unlikely to be of benefit [27]. 


\section{SUMMARY}

Vitamins are several chemically unrelated families of organic substances that cannot be synthesized by humans so we need it as additional supplements in small quantities to prevent disorders of metabolism. Although most multivitamins are safe if taken in the recommended dosage, they are drugs nonetheless, and overdose in small children is always a concern. This can happen from intake of both too many children's multivitamins or from overdose from eating adult supplements that children mistake for candy.

\section{REFERENCES}

1. Bellows, L., \& Moore, R. (2012). Water-soluble vitamins: B-complex and vitamin $C$. Diss. Colorado State University. Libraries.

2. Sassan, P. (2020). David L Burns. Overview of vitamin A. David Seres, Kathleen J Motil. Lisa Kunins.Uptodate. Aug 21, 2020.

https://www.uptodate.com/contents/overview-ofvitamin-

a?search=vit\%20A\%20toxicity\%20in\%20children\&s ource=search_result\&selectedTitle=2 148\&usage_ty pe=default\&display_rank=1\#H15

3. Talarico, V., Barreca, M., Galiano, R., Galati, M. C., \& Raiola, G. (2016). Vitamin D and Risk for Vitamin A Intoxication in an 18-Month-Old Boy. Case reports in pediatrics, 2016.

4. Carazo, A., Macáková, K., Matoušová, K., Krčmová, L. K., Protti, M., \& Mladěnka, P. (2021). Vitamin A update: Forms, sources, kinetics, detection, function, deficiency, therapeutic use and toxicity. Nutrients, 13(5), 1703.

5. Lorenzo, M., Nadeau, M., Harrington, J., \& Gill, P. J. (2020). Refractory hypercalcemia owing to vitamin A toxicity in a 4-year-old boy. CMAJ, 192(25), E671E675.

6. Pazirandeh, S., Lo, C. W., \& Burns, D. L. (2015). Overview of water-soluble vitamins. UpToDate.

7. Bethesda (MD). (2020). LiverTox: Clinical and Research Information on Drug-Induced Liver Injury [Internet]. November https://www.ncbi.nlm.nih.gov/books/NBK548165/

8. Kennedy, D. O. (2016). B vitamins and the brain: mechanisms, dose and efficacy-a review. Nutrients, 8(2), 68.

9. Martini, L., Pecoraro, L., Salvottini, C., Piacentini, G., Atkinson, R., \& Pietrobelli, A. (2020). Appropriate and inappropriate vitamin supplementation in children. Journal of nutritional science, 9.

10. Abdullah, M., Jamil, R. T., \& Attia, F. N. (2021). Vitamin C (ascorbic acid). StatPearls [Internet].

11. Vitamin, C. (2020). Health Professional Fact Sheet. National Institutes of Health. 2020.

12. Yuen, H. W., \& Becker, W. (2021). Iron toxicity. StatPearls [Internet].

13. Farnaghi, F., Hassanian-Moghaddam, H., Zamani, N., Gholami, N., Gachkar, L., \& Yazdi, M. H. (2020).
Vitamin D toxicity in a pediatric toxicological referral center; a cross-sectional study from Iran. $B M C$ pediatrics, 20(1), 1-5.

14. Galior, K., Grebe, S., \& Singh, R. (2018). Development of vitamin D toxicity from overcorrection of vitamin D deficiency: a review of case reports. Nutrients, 10(8), 953.

15. Buyuker, S. M. (2019)."Vitamin D deficiency and toxicity." Eurasian J Med Oncol , 3(1); 1-5.

16. Mark, R. (2020). Vitamin Toxicity Workup. Michael A Miller. Medscape Jun 1 https://www.medscape.com/answers/819426102369/at-what-dose-is-vitamin-d-toxic

17. Vogiatzi, Maria, G. (2014). "Vitamin D supplementation and risk of toxicity in pediatrics: a review of current literature." The Journal of Clinical Endocrinology \& Metabolism, 99(4); 1132-1141.

18. A MORSY, T. O. S. S. O. N., \& D ALANAZI, A. B. D. U. L. L. A. H. (2020). A mini-overview of vitamin E. Journal of the Egyptian Society of Parasitology, 50(2), 247-257.

19. Rizvi, S., Raza, S. T., Faizal Ahmed, A. A., Abbas, S., \& Mahdi, F. (2014). The role of vitamin E in human health and some diseases. Sultan Qaboos University Medical Journal, 14(2), e157.

20. Awuchi, C. G., Igwe, V. S., \& Amagwula, I. O. (2020). Nutritional diseases and nutrient toxicities: A systematic review of the diets and nutrition for prevention and treatment. International Journal of Advanced Academic Research, 6(1), 1-46.

21. Stafford, D. W. (2005). "The vitamin K cycle." Journal of Thrombosis and Haemostasis, 3(8); 1873-1878.

22. SHAFIK, Y. M., \& MORSY, T. A. (2020). MINIOVERVIEW OF VITAMIN K. Journal of the Egyptian Society of Parasitology, 50(3), 522-534.

23. Sassan, P. (2020). David L Burns. Overview of vitamin K. David Seres, Kathleen J Motil. Lisa Kunins.Uptodate. Nov 11, 2020.

https://www.uptodate.com/contents/overview-ofvitamin-

$\mathrm{k}$ ?search $=$ vitamin $\% 20 \mathrm{~K} \&$ source $=$ search_result\&selec tedTitle $=2 \sim 148 \&$ usage_type $=$ default $\&$ display_rank $=1$

24. Mark, R. (2021). Vitamin Toxicity Workup. Michael A Miller. Medscape Oct 20 https://emedicine.medscape.com/article/819426workup

25. Demir, K. (2019). "Comparison of treatment regimens in management of severe hypercalcemia due to vitamin D intoxication in children. "Journal of clinical research in pediatric endocrinology, 11(2); 140.

26. Yuen, Ho-Wang., \& Wenxia, B. (2021). "Iron toxicity." StatPearls [Internet].

27. Christopher, P., Holstege. (2020). Pediatric Iron Toxicity Treatment \& Management. Stephen L Thornton.Medscape Aug 04, 2020. https://emedicine.medscape.com/article/1011689treatment\#d7 